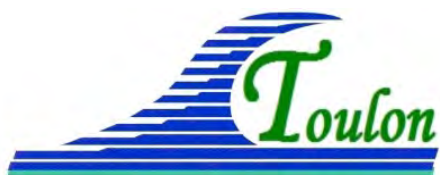

XIV èmes Journées Nationales Génie Côtier - Génie Civil Toulon, 29 juin au $1^{\text {er }}$ juillet 2016

DOI:10.5150/jngcgc.2016.022 (C) Editions Paralia CFL disponible en ligne - http://www.paralia.fr - available online

\title{
Réduire l'érosion des plages lors des tempêtes grâce à des structures immergées : une étude expérimentale comparative en canal à houle
}

\author{
Mathieu GERVAIS ${ }^{1}$, Damien PHAM-VAN-BANG ${ }^{1,2}$, Marissa YATES $^{1,2}$, \\ Vincent VIDAL $^{1,2}$, Michel BENOIT ${ }^{1,3}$
}

1. Université Paris-Est, Laboratoire d'Hydraulique Saint-Venant (LHSV), ENPC, EDFR\&D, Cerema, 6 quai Watier, BP 49, F-78401 Chatou, France. mathieu.gervais@laposte.net

2. Cerema, DTecEMF, 134 rue de Beauvais, F-60280 Margny-les-Compiègne, France. damien.pham-van-bang@cerema.fr ; marissa.yates-michelin@cerema.fr

3. Institut de Recherche sur les Phénomènes Hors-Equilibre (IRPHE), UMR 7342 (CNRS, Aix-Marseille Université, Ecole Centrale Marseille), 49 rue Frédéric JoliotCurie, BP 146, F-13384 Marseille Cedex 13, France. benoit@irphe.univ-mrs.fr

\section{Résumé :}

L'objectif de cette étude est de caractériser et d'optimiser l'efficacité de différentes structures immergées parallèles à la côte (brise-lame immergé, boudin simulant un géotube, dalle anti-affouillement) pour réduire l'érosion des plages. Des modèles réduits de plages avec des structures immergées (échelle 1/10) et une procédure expérimentale sont proposés afin de comparer l'efficacité des structures. Pour une hauteur de submersion constante et un scénario de tempête donné, les effets de la forme et de la distance au trait de côte des structures sont comparés. La sensibilité des résultats aux scénarii de tempête ou au profil initial de la plage est par ailleurs analysée.

Mots-clés : Erosion de tempête, Brise-lames immergés, Boudins géotextiles, Ingénierie côtière, Travaux maritimes, Modèle physique, Morphodynamique de plage, Résilience.

\begin{abstract}
:
This study aims to characterize and optimize the efficiency of different submerged structures parallel to coasts (submerged breakwater, geotextile tube, anti-scouring slab) to reduce beach erosion. Several reduced-scaled models of beaches with structures (1/10) and an experimental protocol are proposed to compare the efficiency of structures. With a constant submerged depth and for the same storm scenario, the effects of structure's shape and distance from the coastline are compared. The sensitivity of results to the particular storm scenario or initial profile of the beach is then evaluated.
\end{abstract}




\section{Thème 2 - Dynamique sédimentaire}

\section{Introduction}

Les processus gouvernant l'évolution des plages s'effectuent à de multiples échelles spatiales (celle du grain, de la barre d'avant-côte ou du profil de plage) et temporelles (échelle d'une vague, d'une tempête ou d'une année).

Si une plage se remanie sans cesse, il est toutefois possible de considérer qu'à l'échelle saisonnière les tempêtes constituent des événements majeurs dans cette dynamique (cf. CASTELLE et al., 2015 ; BALOUIN et al., 2015). En effet, les tempêtes occasionnent des pertes locales de sédiments (recul des barres et/ou creusement de la plage). Différentes solutions ont donc été imaginées pour y remédier. Elles consistent soit à compenser ces pertes de sédiments (rechargement de plages), soit à réduire l'intensité des phénomènes érosifs par des structures. Les solutions immergées, de type brise-lame ou boudin de géotube, sont aujourd'hui privilégiées car elles permettent une bonne circulation des masses d'eau (donc une meilleure qualité des eaux), allongent la durabilité des rechargements de plage, et réduisent les effets des tempêtes, notamment l'export de sable vers le large ou longitudinalement vers des sites voisins (cf. BALOUIN et al., 2015). Leur impact sur le paysage côtier est aussi bien moindre en comparaison des digues émergées.

Pour les structures immergées, il reste néanmoins un besoin de connaissances pour établir les règles de conception ou d'optimisation en vue de leur implémentation sur un site spécifique. En effet, même si les outils de calcul incluent les récentes avancées en morphodynamique côtière, par exemple, la dynamique liée aux ondes longues pour l'érosion dunaire (ROELVINK et al., 2009), ou encore l'asymétrie des vagues / forme des brisants sur le mouvement cross-shore des barres d'avant-côte (DUBARBIER et al. 2015), leur application tenant compte des spécificités de chaque site reste difficile (CALLAGHAN et al., 2013). En outre, les différents retours d'expérience sur des structures immergées réelles (notamment dans le cadre du projet européen DELOS, BURCHARTH et al., 2007) n’ont pas permis d'expliquer les raisons d'une réussite ou d'un échec de l'aménagement du fait de la disparité des structures et des sites testés.

Cette étude propose de quantifier les performances de différentes structures immergées (brise lame immergé -BLI, boudin simulant un géotube, dalle anti-affouillement) pour protéger une plage en considérant un profil initial et un scénario de tempête donné. Les effets de la forme et des dimensions des structures sont testés pour une hauteur de submersion constante. Le modèle physique et la procédure expérimentale pour tester différentes structures est tout d'abord présenté (Partie 2), puis les résultats des essais sont analysés dans la partie 3. Une conclusion et des recommandations pour les futurs tests sont proposées en partie 4 .

\section{Matériel et méthodes}

L'expérimentation est conduite dans un canal à houle au LHSV à Chatou (78). L'état initial de la plage (avant d'inclure une structure immergée ou d'imposer un scénario de 


\section{XIVìmes Journées Nationales Génie Côtier - Génie Civil \\ Toulon, 29 juin au $1^{\text {er }}$ juillet 2016}

tempête) est préparé selon une méthodologie similaire à celle proposée par GRASSO et al. (2009) au LEGI pour étudier les profils cross-shore de plages à l'équilibre.

Dans notre étude, le profil cross-shore de plage à l'équilibre est obtenu à partir d'un climat (calme) de vagues. Il sert d'état de référence à partir duquel est mesurée l'évolution de la plage suite à une tempête $(T)$, en présence ou non d'une structure de protection. La procédure diffère un peu de la méthode de GRASSO et al. (2009) afin d’obtenir plus rapidement notre profil initial et son état d'équilibre avec une structure.

\subsection{La configuration expérimentale et le choix du matériau sédimentaire}

Le canal à houle $\mathrm{n}^{\circ} 12$ ayant pour dimension $36 \times 0,6 \times 0,84 \mathrm{~m}$ est utilisé pour cette modélisation de plage à l'échelle 1/10 (similitude de Froude). L'utilisation d'un sable léger en PMMA (densité $=1,19$ et $d_{50}=0,540 \mathrm{~mm}$ ) permet également de respecter les similitudes de Shields et de Rouse, sur la mobilité et la suspension des sédiments respectivement. Selon les études de GRASSO et al. (2009), la plage modélisée est de type intermédiaire, avec une pente moyenne de 2,7\% où, comme en nature, peut apparaitre une barre d'avant-côte à des distances réalistes de la plage.

Le canal est équipé d'un batteur à houle (type piston) asservi pour produire une série de vagues irrégulières (ou run, de durée 30 minutes) à paramètres statistiques $\left(H_{s}, T_{p}\right.$. contrôlés respectant un spectre de type JONSWAP (avec un facteur de pic $\gamma=3,3$ ). Les caractéristiques des vagues sont mesurées tout au long de leur propagation vers la plage en quatorze points (figure 1A). L'évolution du profil de la plage est suivie après chaque run par sonar acoustique et/ou relevé manuel.

\subsection{Les structures immergées}

Trois types de structures submergées à différentes distances de la plage sont testées (figure 1A) : elles sont toutes lisses (légèrement rugueuses), non-poreuses, avec une profondeur de submersion de la crête constante (-1 m à l'échelle nature).

Les deux premières géométries sont de formes classiques : un trapèze et une ellipse simulant un brise-lame (BLI) et un géotube. Leurs dimensions sont à l'échelle 1/10 des structures réelles (figure 1B). Pour tester l'effet de la distance à la plage (à égale hauteur de submersion), deux tailles de structures ont été considérées. Le troisième type de structure est inspiré de dalles anti-affouillement posées sur le fond, ou d'un banc de grès (beachrock) aggloméré en surface. Deux longueurs de dalles ont été testées.

Dans nos conditions, les 6 structures testées occupant toute la largeur du canal correspondent sur le terrain à des structures infiniment étendues dans la direction parallèle à la plage. Seules les dalles sont libres et susceptibles de s'enfoncer avec l'affouillement, les deux premières structures étant bloquées contre les parois du canal. Le courant de retour/compensation passe essentiellement au-dessus de l'ouvrage. 


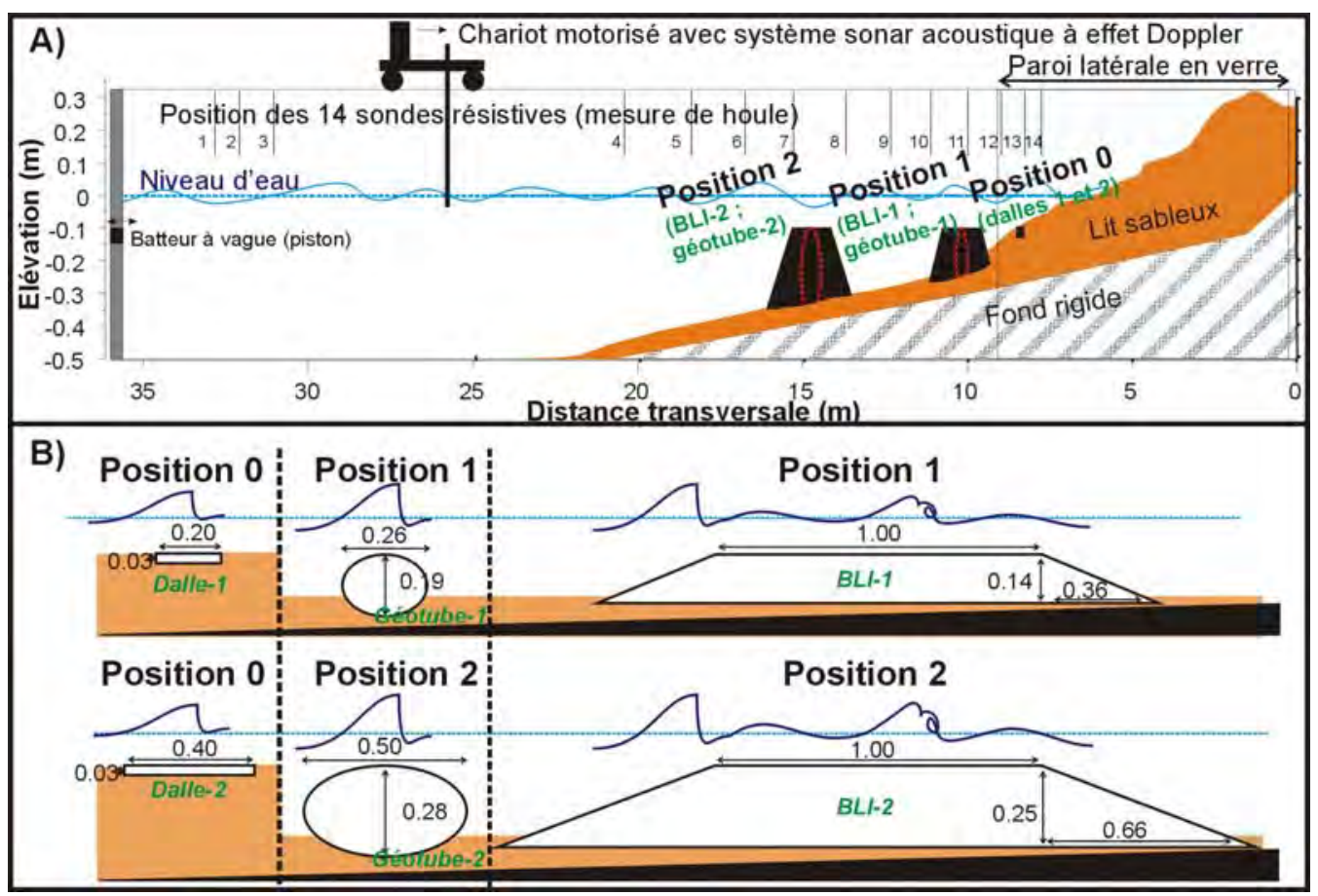

Figure 1. A) Schéma du dispositif expérimental et B) dessins techniques des 6 structures.

\subsection{Les climats pré-tempête et les scénarii de tempête}

Pour la réalisation de profils initiaux ou d'état de référence pré-tempête de plage, avec ou sans structure, trois climats de vagues correspondant à une houle modérée ont été retenus. Le premier climat "calme $0 "\left(\mathrm{H}_{\mathrm{s}}=0,145 \mathrm{~m} ; \mathrm{T}_{\mathrm{p}}=2 \mathrm{~s}\right.$ à l'échelle modèle, paramètres mesurés au début du profil sableux) a été considéré afin de tester, avec succès, la reproductibilité des résultats de GRASSO et al. (2009) dans nos conditions.

Le second climat "calme 1" $\left(\mathrm{H}_{\mathrm{s}}=0,12 \mathrm{~m} ; \mathrm{T}_{\mathrm{p}}=1,6 \mathrm{~s}\right)$ est très proche du climat $\mathrm{E}$ détaillé par GRASSO et al. (2009) : il correspond à une houle courte, typique des conditions "moyennes" de vagues dans une mer fermée comme la Méditerranée, et sert aussi à adapter l'équilibre obtenu sans structure aux conditions avec l'une des six structures testées. Pour le climat "calme 1", tous les profils obtenus sans structure sont quasiidentiques. Et afin de tester l'influence du profil pré-tempête, un troisième climat "calme 2" ( $\mathrm{H}_{\mathrm{s}}=0,123 \mathrm{~m}$; $\left.\mathrm{T}_{\mathrm{p}}=2,6 \mathrm{~s}\right)$ a été considéré, représentant un site plus océanique avec des vagues à plus forte période en conditions de non tempête.

Les profils d'équilibre (obtenus à partir de l'un de ces trois climats pré-tempête), avec ou sans structures, sont finalement soumis à un évènement de tempête, selon le scénario $T 1$ ou T2. La figure 2 présente l'évolution temporelle des paramètres de vague $\left(\mathrm{H}_{\mathrm{s}} ; \mathrm{T}_{\mathrm{p}}\right)$ lors des deux scénarii de tempête. Le scénario $T 1$ est représentatif d'un cas méditerranéen et a été imposé sur les plages résultant du climat "calme 1" pré-tempête avec ou sans structures. Le scénario $T 2$ correspond à une tempête $T 1$ plus longue 


\section{XIV èmes Journées Nationales Génie Côtier - Génie Civil \\ Toulon, 29 juin au $1^{\text {er }}$ juillet 2016}

(durées de pic et de tombant deux fois plus longues) afin de tester l'influence du tombant sur le profil final, post-tempête. Ce scénario a uniquement été testé dans deux cas : sans ouvrage, puis avec un ouvrage particulier (géotube, position 1).

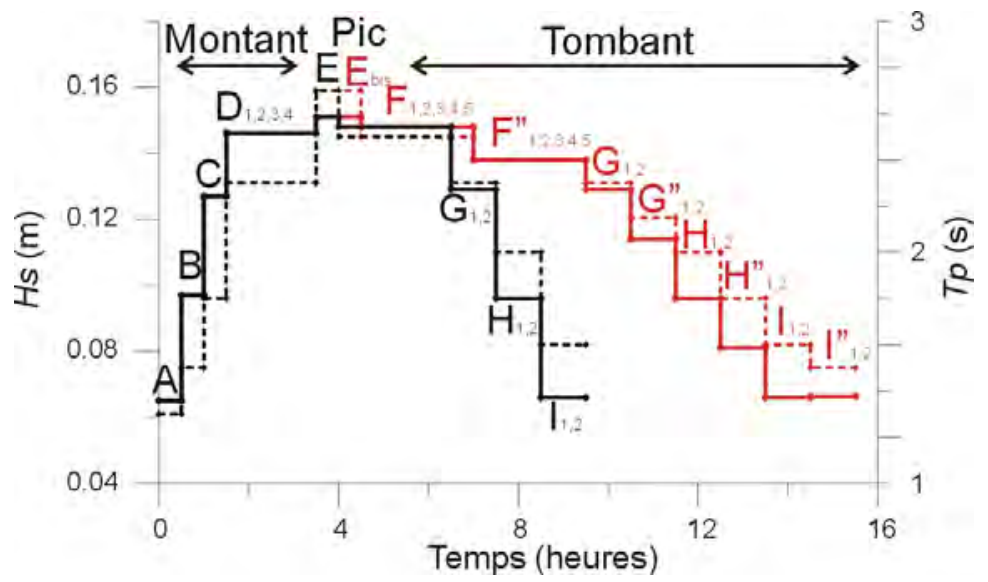

Figure 2. Evolutions temporelles de $H_{s}$ (trait plein) et $T_{p}$ (pointillés) mesurées au début du profil sableux (sonde 4) pour les scénarios de tempête T1 (en noir) et T2 (en rouge).

\section{Résultats et analyses}

\subsection{Profils d'équilibre sous différents climats de pré-tempête}

La comparaison des profils obtenus pour les trois climats qui ont servi à l'obtention d'états de référence permettent de conclure quant à la bonne reproductibilité des résultats de GRASSO et al. (2009) d'une part, et à la très bonne répétabilité des profils pour nos conditions de tests. L'évolution haute fréquence montre des temps d'adaptation différents selon le profil initial et des effets importants de l'ajustement du profil, ainsi que des rétroactions intéressantes dans la dynamique barre/plage.

\subsection{L'adaptation du profil à une structure en conditions pré-tempête}

L'introduction d'une structure sur le profil à l'équilibre induit inévitablement une perturbation dans le système, à l'exception du cas des dalles qui affleurent la surface du fond marin. Donc il est nécessaire d'imposer un climat de vague pré-tempête afin de laisser le système (avec sa structure) s’auto-équilibrer. Ceci est assez rapide (environ 2 à 3h sur le modèle) et se concrétise par une légère accumulation ou érosion (ou affouillement) de part et d'autre des géotubes / BLI, avec des modifications du profil près de la plage.

\subsection{L'impact d'une tempête selon les 6 designs de protection}

La figure 3A-G présente les évolutions bathymétriques clefs au cours de la tempête $T 1$ sans et avec l'un des 6 ouvrages de protection testés. L'érosion des plages étant d'autant plus réduite que l'énergie des vagues est atténuée, les résultats mettent en évidence 


\section{Thème 2 - Dynamique sédimentaire}

qu'une structure de type brise-lame est plus efficace qu'une structure de type géotube, elle-même plus efficace qu'une structure de type dalles.

Les dalles ne produisent presque pas d'effet en s'enfonçant avec le lit près de la plage. Les géotubes et brise-lames ne suppriment pas le processus d'érosion, mais en réduisent significativement l'importance. Et si la solution brise-lame réduit davantage l'érosion que la solution géotube, elle est plus exposée à des problèmes d'affouillement.

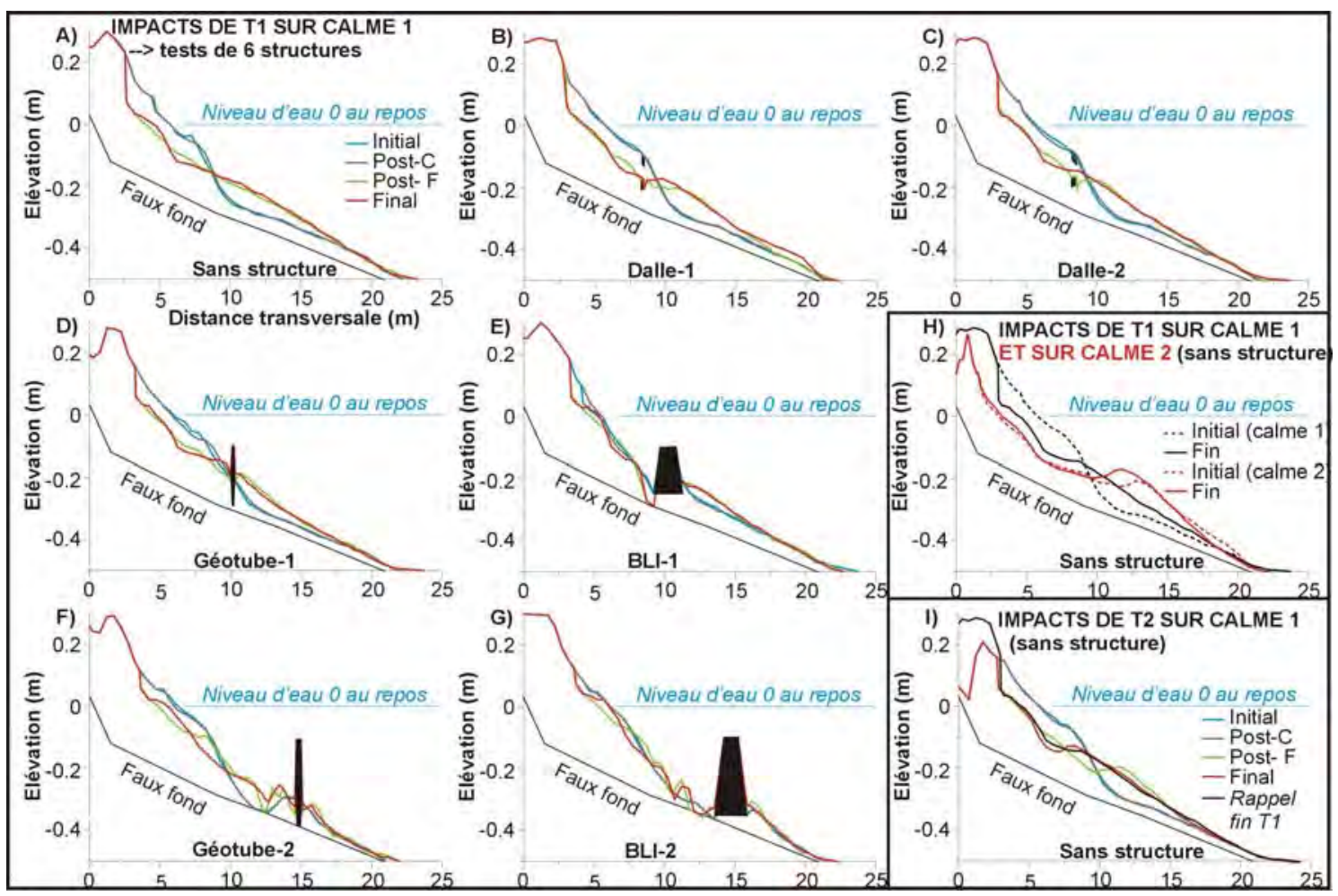

Figure 3. Evolutions clefs du profil lors des tempêtes: les cas A) à G) présentent les impacts de $T 1$ sur une plage issue du calme 1 avec les différentes structures; le cas $H$ ) les impacts résultant de $T 1$ sur le profil d'équilibre calme 1 (en noir) en comparaison de calme 2 (en rouge) sans structure. Le cas I) l'évolution avec T2 en comparaison de l'état final T1 dans la configuration de test sans structure.

Les processus d'affouillement sont manifestes dès la phase de rééquilibrage (cf. 3.2) consécutive à l'insertion de la structure en conditions de pré-tempête. Toutefois ils sont faibles. Ils sont en revanche fortement amplifiés lors de la tempête, en atteignant un maximum au pic de cette dernière et en diminuant par comblement lors du tombant.

La distance de la structure à la plage (pour une même hauteur de submersion) affecte les ordres de grandeur sur les bilans sédimentaires (figure 4) sans inverser le classement sur l'efficacité des structures : le brise-lame réduit plus fortement l'érosion que le géotube. Plus la structure est éloignée et large, plus les hauteurs de vagues sont atténuées et 


\section{XIV ${ }^{\text {èmes }}$ Journées Nationales Génie Côtier - Génie Civil \\ Toulon, 29 juin au $1^{\text {er }}$ juillet 2016}

l'érosion réduite. En revanche, cela amène à des super-structures très volumineuses et donc très couteuses, un compromis est donc nécessaire.

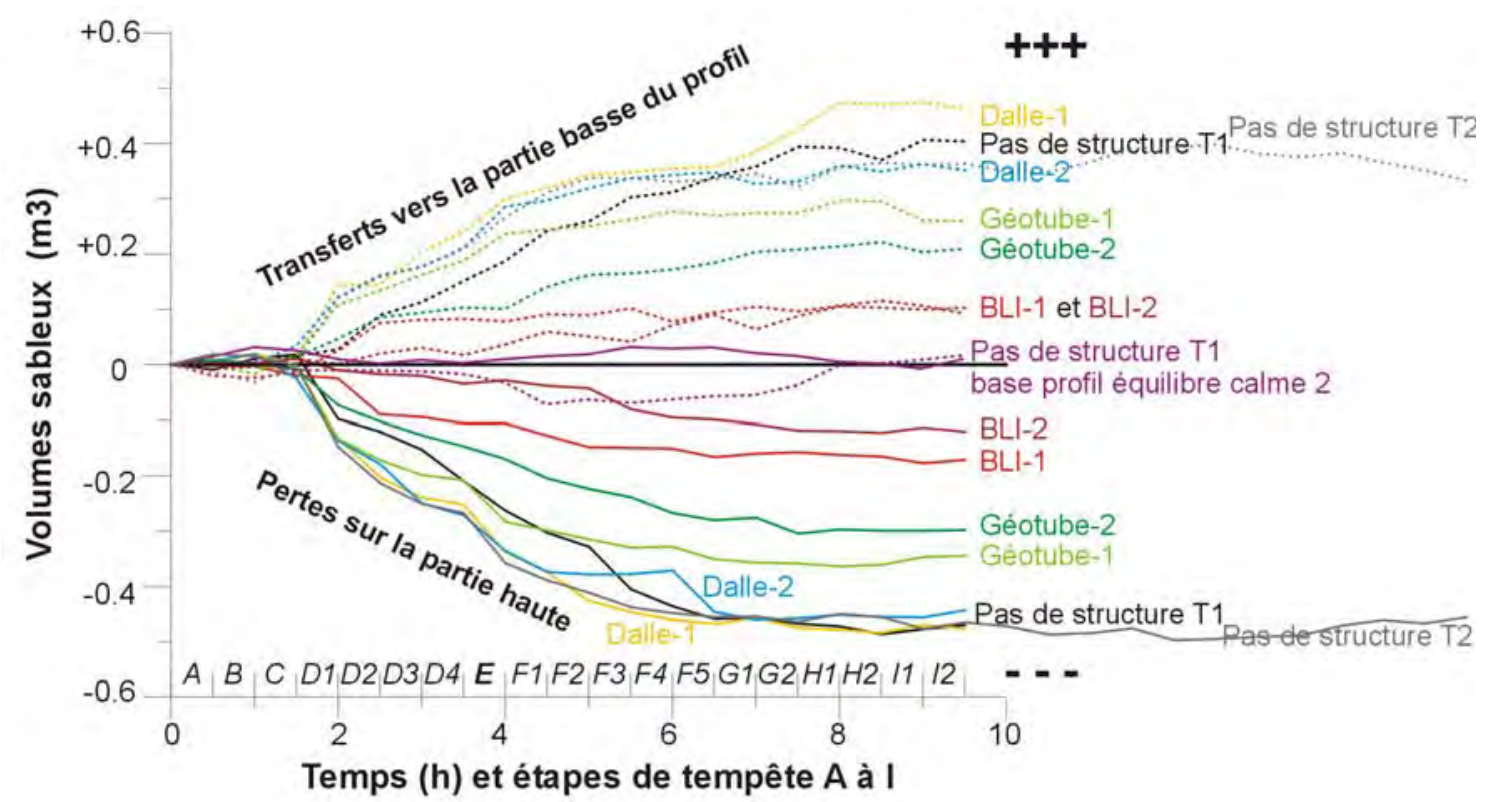

Figure 4. Variation temporelle du volume de sable entre la partie haute (trait plein) et basse (pointillé) du profil de plage (limite fixe distance cross-shore $X=9,5 \mathrm{~m} ; c f$. figure 3), pour les expériences de tempête T1 avec et sans ouvrages de protection (base calme 1), le cas de T2 sans ouvrage, et le cas de T1 sur le profil "océanique" calme 2.

\subsection{L'importance de la morphologie pré-tempête et du site}

Pour le scénario de tempête $T 1$, l'effet du profil pré-tempête (calme 1 et calme 2) sur le profil final (post-tempête) est très important (figure $3 \mathrm{H}$ ). Dans le cas de calme 2, la présence d'une barre modifie significativement les caractéristiques des vagues sur l'avant côte. Le profil calme 2 est quasi-insensible à la tempête $T 1$, seule la barre oscille. Cela s'explique par la forte similarité des conditions calme 2 avec celles des conditions du pic de tempête en terme de période des vagues $\left(\mathrm{H}_{\mathrm{s}}=0,123 \mathrm{~m}, \mathrm{~T}_{\mathrm{p}}=2,6 \mathrm{~s}\right.$ pour calme 2 alors que $\mathrm{H}_{\mathrm{s}}=0,151 \mathrm{~m}$ et $\mathrm{T}_{\mathrm{p}}=2,7 \mathrm{~s}$ lors du pic $\mathrm{E}$ de $T 1 / \mathrm{T} 2$ )

\subsection{L'importance du scénario de tempête}

Pour le profil calme 1, la durée doublée de la tempête (du pic et du tombant ; 49h au lieu de 30h au total en nature) modifie peu le bilan sédimentaire (figure 4). Dans ces conditions, il est observé que la plage s’ajuste surtout lors du montant, au début des conditions de fortes vagues: ni la durée du pic ni celle du tombant ne semble modifier significativement l'état final. 


\section{Conclusions et remarques-perspectives}

Les études d'amortissement de vagues par des structures restent souvent réduites à des mesures de coefficients de transmission en l'absence d'érosion, et les études sur fonds érodables à des mesures de profils à l'équilibre en absence de structure sur l'avant-côte. Cette nouvelle étude permet de combler le manque de résultats sur modèles réduits de structures dans un environnement érodable. Elle s'appuie sur un strict respect des conditions de similitude pour un écoulement à surface libre et le transport sédimentaire. La méthodologie se base sur l'obtention d'un profil à l'équilibre, aménagé ou non d'une structure d'amortissement des vagues, puis soumis à des scénarii de tempêtes. Les résultats pour ces différentes conditions (profils initiaux de plage, solutions d'aménagements, scénario de tempête) sont suffisamment répétables pour intercomparer les résultats et envisager une optimisation dans la conception des structures.

Pour les essais menés, les brise-lames sont à la fois plus efficaces vis-à-vis de l'érosion grâce à un processus dissipatif plus efficace, mais aussi plus vulnérables (peut-être en raison de cela) vis-à-vis de l'affouillement que les géotubes et les dalles. L'accès aux champs de vitesses à proximité de la structure permettrait de mieux détailler ce processus dissipatif. La méthodologie d'étude serait transposable au cas des structures poreuses, à condition de vérifier la similitude sur la perméabilité. Dans les conditions retenues pour cette étude, l'efficacité des structures testées semble dépendre du profil initial pré-tempête et, dans une moindre mesure, du scénario de tempête pour des puissances de vagues comparables. Il conviendrait d'étudier plus précisément ces effets morphodynamiques dans les futures études. Enfin, cette étude en canal à houle impose des restrictions fortes sur l'utilisation des résultats puisque les processus tridimensionnels sont implicitement négligés.

\section{Remerciements}

Les auteurs remercient la société Géocorail SAS ® d'avoir financé cette étude.

\section{Références bibliographiques}

BALOUIN Y., LONGUEVILLE F., COLOMBET Y. (2015). Video Monitoring of soft coastal defenses at the Lido of Sete, France. 3rd Coastal and Maritime Mediterranean Conference, Ferrara, Italia, pp 191-196. http://dx.doi.org/10.5150/cmcm.2015.038

BURCHARTH H.F., HAWKINS S.J., ZANUTTIGH B., LAMBERTI A. (2007). Environmental design guidelines for low crested coastal structures. Livre recueil du projet DELOS, Edition Elsevier Science, 393 p.

CASTELLE B., MARIEU V., BUJAN S., SPLINTER K.D., ROBINET A., SENECHAL N., FERREIRA S. (2015). Impact of the winter 2013-2014 series of severe Western Europe storms on a double-barred sandy coast: Beach and dune erosion and 


\section{XIV ${ }^{\text {èmes }}$ Journées Nationales Génie Côtier - Génie Civil \\ Toulon, 29 juin au $1^{\text {er }}$ juillet 2016}

megacusp embayments. Geomorphology, Vol. 238, pp 135-148. http://dx.doi.org/10.1016/j.geomorph.2015.03.006

DUBARBIER B., CASTELLE B., MARIEU V., RUESSINK G. (2015). Process-based modeling of cross-shore sandbar behavior. Coastal Engineering, Vol 95, pp. 35-50. http://dx.doi.org/10.1016/j.coastaleng.2014.09.004

GRASSO F, MICHALLET H., BARTHELEMY E., CERTAIN R. (2009). Physical modeling of intermediate cross-shore beach morphology: transient and equilibrium states. Journal of Geophysical Research, Vol. 114(C9). http://dx.doi.org/10.1029/2009JC005308 ROELVINK D., RENIERS A., VAN DONGEREN A., VAN THIEL DE VRIES J., MCCALL R., LESCINSKI J. (2009). Modelling storm impacts on beaches, dunes and barrier islands. Coastal Engineering, Vol. 56(11-12), pp 1133-1152. http://dx.doi.org/10.1016/j.coastaleng.2009.08.006 
Thème 2 - Dynamique sédimentaire 